DSF $-07 / 2006$

\title{
TEACHING THEORETICAL PHYSICS: THE CASES OF ENRICO FERMI AND ETTORE MAJORANA
}

\author{
A. DE GREGORIO AND S. ESPOSITO
}

\begin{abstract}
We report on theoretical courses by Fermi and Majorana, giving evidence of the first appearance and further development of Quantum Mechanics teaching in Italy. On the basis of original documents, we make a comparison between Fermi's and Majorana's approaches. A detailed analysis is carried out of Fermi's course on Theoretical Physics attended by Majorana in 1927-28. Three (previously unknown) programs on advanced Physics courses submitted by Majorana to the University of Rome between 1933 and 1936 and the course he held in Naples in 1938 complete our analysis: Fermi's phenomenological approach resounded in Majorana, who however combined it with a deeper theoretical approach, closer to the modern way of presenting Quantum Mechanics.
\end{abstract}

\section{INTRODUCTION}

The old-dated tradition going back to Galileo Galilei made the study of the Physical Sciences in Italy essentially meant as experimental still in the 1920s, though the theoretical aspect was not thoroughly excluded from the scientific debate. In this respect, the case is emblematic of Enrico Fermi, whose thesis for a degree in Physics at the University of Pisa, "according to tradition, should be in Experimental Physics;" [1. Then, it is not surprising that, in Italy, no stable chair of Theoretical Physics was yet established at that time nor that the scientific debate on the emergent novel Quantum Mechanics was still languishing, all the more so if one compares it with the fruitful discussions abroad.

This outline started changing with the first competition for a stable chair of Theoretical Physics in 1926: the young Enrico Fermi then got a position as a professor in Rome and strove to introduce the novel concepts of Quantum Mechanics into advanced academic courses. In the following years, a whole generation of novelminded physicists (both theoreticians and experimentalists) was formed by Fermi and his coworkers. Their relevant contributions extended to several areas of Physics, in Italy as well as in other countries. It is then of key importance to analyze Fermi's courses on Theoretical Physics. This analysis is made possible by some record books reporting the content of Fermi's lectures in Rome. Together with the textbooks on Theoretical Physics Fermi wrote in those same years, these documents lead to a clear and comprehensive picture of Fermi's courses.

Most of the well versed experimentalists who worked in close collaboration with Fermi in the 1930s had attended Fermi's theoretical courses. On the other hand, most of the well versed theoreticians who collaborated with Fermi's group in the Thirties, like Gian Carlo Wick and Giovannino Gentile, had not attended university courses in Rome. One relevant exception was Ettore Majorana, who followed 
Fermi's course on Theoretical Physics together with Segrè and Edoardo Amaldi in 1928.

Majorana contributed significantly to some theoretical aspects underlying the researches performed under the supervision of Fermi and in general by Fermi's associates. However, we will focus here on Majorana's own progress concerning the basics (and the applications) of Quantum Mechanics. Majorana's lectures on Theoretical Physics very effectively give evidence of this progress. He delivered them in 1938, when he obtained a position as a full professor in Naples (just before he mysteriously disappeared): that was the first chair of Theoretical Physics ever established in Italy since the one Fermi obtained in 1927. A recent analysis 2] showed that Majorana's 1938 course was very innovative for that time.

Before then, Majorana submitted three programs concerning advanced Physics courses he would have held at the Rome University between 1933 and 1937. Through the recent retrieval of these programs, whose existence was unexpected, we shall follow the evolution of Majorana's approach to Quantum Mechanics teaching. Further, we shall point out how Fermi's and Majorana's courses relate to each other, showing conceptual differences as well as impressive similarities that denote particularly relevant teaching strategies.

In the following section, we give an account of Fermi's lectures on Theoretical Physics in Rome. In Sect. 3, we point out the main stages leading Majorana to his professorship at the University of Naples and describe the relevant features of his lectures. The progress of Majorana's approach to Quantum Mechanics, from Fermi's lectures to his own lectures on Theoretical Physics, is dealt with in Sect. 4. In the subsequent section, the common traits of Fermi's and Majorana's courses are discussed. In Sect. 6, we put forward some speculations on the missing notes of the first four lectures by Majorana, also discussing his teaching strategies in a broader perspective. We give our conclusions in Sect. 7. The programs of the three courses presented by Majorana at the University of Rome from 1933 to 1936 are reported in the Appendix, together with a list of the topics Fermi lectured on in his 1927-28 course in Theoretical Physics.

\section{Fermi's lectures on Theoretical Physics}

2.1. Professor in Rome. On December 22, 1924 the Faculty of Sciences at the University of Rome debated about the institution of a chair of Theoretical Physics, tenaciously promoted by Orso M. Corbino, director of the Institute of Physics and influential politician. The chair was formally claimed on April 27, 1925. The board of examiners met in November 1926 and proclaimed winners Enrico Fermi, Enrico Persico and Aldo Pontremoli, respectively. Fermi was formally appointed as a professor of Theoretical Physics at the Royal Institute of Physics in Rome starting from January 1, 1927. Persico and Pontremoli were appointed at the Universities of Florence and Milan, respectively.

With the support of Corbino, Fermi had already taught at the University of Rome, lecturing on Mathematics to chemists and naturalists in 1923-24; then he spent two more academic years lecturing on Mathematical Physics in Florence. On 
January 20, 1927 he gave his first lecture on Theoretical Physics in Rome ${ }^{1}$. Noticeably, his course was annotated by Carlo Dei and Leonardo Martinozzi and then published [3] the record book reporting the content of Fermi's lectures is available as well, kept at the Archives of the University of Rome "La Sapienza." A strict correspondence can be established between the topics reported in the handbook and those that Fermi actually lectured on in 1927.

An even deeper insight into Fermi's lectures on Theoretical Physics is provided by the book he published in the following year [4. The 1927-28 teacher's record book is now available as well, and we shall see below how strong the correspondence is between the content of the lectures Fermi held in 1927-28 and the book he published in 1928. For the moment, we only mention that Ettore Majorana together with Edoardo Amaldi and Emilio Segrè followed precisely this course. They switched from Engineering to Physics just following the appeals by Corbino, who was striving to build up a forefront school of Physics upon Fermi.

Amaldi moved to Physics on November 9, 1927, at least formally, followed by Segrè on February $8,1928^{2}$; both took their exams in Theoretical Physics in July 1928 like Majorana, though the latter was still a student in Engineering and obtained permission to move to Physics only on the following November $19 .{ }^{3}$ Majorana and Amaldi also enjoyed another course held by Fermi, who also lectured on Earth Physics in 1928-29. They took the corresponding exam on June 27, 1929, just few days before they graduated on July 6 .

2.2. The 1927-28 course in Theoretical Physics. An outline of the main points of the course in Theoretical Physics held by Fermi in 1927-28 can be easily drawn from his teacher record book. Fermi gave sixty-five lectures (the last six ones aimed at summarizing the whole course). He started with the Kinetic Theory of gases and the basic elements of Statistical Mechanics: in a first set of lectures Fermi expounded classical topics such as the dependence of pressure on the molecular kinetic energy, mean free path, equipartition of the energy, the phase space, the Boltzmann distribution law and the Maxwell velocity distribution. A second set dealt with electromagnetism: electromagnetic perturbation, the Poynting vector, the electronic theory of dispersion, radiation theory. Fermi then passed to introduce the foundations of the atomic theory, lecturing on the electron, the radioactive transmutation, Rutherford's atomic model, light quanta, the Compton effect, and a short mention to Bohr's atom and energy levels. Therefore the 'core' of Fermi's course took shape, taking twenty lectures on Bohr's model of the atom. Energy levels, the Rydberg constant, stationary states, the Sommerfeld conditions, the fine structure, the correspondence principle, selection rules, the Bohr magneton, spatial quantization, the Zeeman and the Stark effects are some of the topics dealt with in lectures from n. 28 to n. 47.

\footnotetext{
${ }^{1}$ It should be noted that although Fermi's chair was the first one ever established in Italy, his course was not the first one ever given on Theoretical Physics. As an example, we only mention the case of Antonio Carrelli, who regularly lectured on Theoretical Physics in Naples since 1924.

${ }^{2}$ This information comes from the Archives of the University of Rome "La Sapienza", where the reports of the Board of the Faculty of Science are kept. It is intriguing that, according to Amaldi and Segrè, they passed from Engineering to Physics just in the reverse order [5], 6].

${ }^{3}$ We point out that the only exam Majorana took in 1928 was that in Theoretical Physics, according to his scholastic career report. His mark was expressed in hundredths, as accustomed for engineers, while Amaldi and Segrè's in thirtieths [5], 6].
} 
One striking feature emerges here: apart from a fleeting mention of the "quantum theory of the hydrogen atom" in lecture n.46, all these lectures rested on the framework of Old Quantum Theory. The same holds for the remainder of Fermi's course, essentially devoted to the spectra of alkali metals, alkaline earth metals and atoms with three valence electrons.

Another trait worth remarking is that relativity was not mentioned among the issues of Fermi's 1927-28 course. The same holds for his 1926-27 course where, although the Compton effect was explicitly reported, the relativistic kinematics was not discussed. Therefore, one might even wonder how Fermi could lecture on the Compton effect at all. Remarkably, he carried out his calculations neglecting terms of order greater than the first in the difference $\nu-\nu^{\prime}$ of the light frequency and then specified:

The final formula is strictly exact. That might appear a nonsense, since we neglected such terms as $(\mathrm{d} \lambda)^{2}$. However, it should be noted that we had already made another approximation when we expressed the momentum of the electron as $m v$ instead of $m v / \sqrt{1-\beta^{2}}$ therefore neglecting relativity. Now, the curious affair is that an opposite error creeps in this way, so that the formula above turns out to be strictly exact instead of being an approximation (see Ref. [3], pp. 81-82).

The strict correspondence between the topics reported in the 1927-28 record book and those explained in the 1928 book in Ref. 4 can be readily realized by merely comparing the content of the first nine lectures ranging from November 15 to December 3, 1927 with the titles of the sections in the first chapter of Fermi's book. We give both in Table 1. The whole of the contents of Fermi's second course on Theoretical Physics is instead reported in the Appendix. Given such a correspondence between the lectures and the book in Ref. [4, we can group the lectures into six sets: each relates to one of the first six chapters of Ref. 4. as illustrated in Table 2. The correspondence is very strict in the case of the first two chapters, while slight differences come out starting from the third chapter: sometimes the order of the topics of the course is altered in comparison with the book, and in the latter several arguments might receive a somewhat more detailed treatment.

\section{EtTore MAjorana: From Fermi's COURSES TO PROFESSORShip}

Ettore Majorana began to frequent the Institute of Physics at the University of Rome at the end of 1927 or in the first months of the following year [5], 6, 7], 8]. It is very likely that the first course he attended at the Institute of Physics was precisely the one in Theoretical Physics given by Fermi and discussed in the previous section. Some indirect records of this can be traced in Majorana's "Volumetti" 9] ("Booklets"), which also contain several issues probably discussed by Fermi in his course on Earth Physics. Since no lecture notes of Fermi's courses followed by Majorana are known, we can only compare what is reported in the Volumetti with Fermi's book 4. In this respect, it is noteworthy that the approach to Quantum Mechanics contained in the Volumetti substantially differed from Fermi's one. That denotes an autonomous work by the student Majorana, who was particularly impressed by the book on Group Theory and Quantum Mechanics 10 Hermann Weyl published at the end of 1928 (see the analysis in Ref. [2]). Nevertheless, the 


\begin{tabular}{|c|c|c|c|}
\hline & Fermi course $(1927-28)$ & & Fermi book (1928) \\
\hline I) & $\begin{array}{l}\text { Interactions between molecules. } \\
\text { Solids and fluids in the framework } \\
\text { of the kinetic theory. }\end{array}$ & 1. & Molecular interactions. \\
\hline II) & $\begin{array}{l}\text { Dependence of pressure upon } \\
\text { molecular kinetic energy. }\end{array}$ & 2. & $\begin{array}{l}\text { Molecular mobility in solids } \\
\text { and fluids. }\end{array}$ \\
\hline III) & Mean free path. Vapor rays. & & \\
\hline IV) & $\begin{array}{l}\text { Kinetic energy in general coordinates. } \\
\text { The theorem of equipartition } \\
\text { of energy (without proof). }\end{array}$ & $\begin{array}{l}3 . \\
4 .\end{array}$ & $\begin{array}{l}\text { Kinetic theory of ideal gases. } \\
\text { Mean free path. }\end{array}$ \\
\hline V) & Phase space. & & \\
\hline VI) & $\begin{array}{l}\text { Evaluation of the probability of } \\
\text { distribution. }\end{array}$ & 5 . & Equipartition of energy. \\
\hline VII) & The Boltzmann distribution law. & 6. & Boltzmann statistical \\
\hline VIII) & $\begin{array}{l}\text { Proof of the principle of } \\
\text { equipartition of the energy. }\end{array}$ & & distribution. \\
\hline IX) & The Maxwell law. & 7. & The Maxwell law. \\
\hline
\end{tabular}

TABLE 1. A comparison between the topics covered in the first nine lectures of Fermi's 1927-28 course, and the sections in the first chapter of his book (Ref. 4]).

\begin{tabular}{|ll|ll|}
\hline \hline \multicolumn{2}{|c|}{ Sets of lectures } & \multicolumn{2}{c|}{ Chapters } \\
\hline \hline n. 1-n. 9: & Nov. 15 - Dec. 3 & I & Kinetic theory of gases \\
\hline n. 10 - n. 19: & Dec. 6 - Jan. 14 & II & Electromagnetic theory of light \\
\hline n. 20 - n. 23: & Jan. 17 - Jan. 24 & III & Electric particles \\
\hline n. 24- n. 27: & Jan. 26 - Feb. 2 & IV & $\begin{array}{l}\text { Energy exchange between } \\
\text { light and matter }\end{array}$ \\
\hline n. 28 - n. 47: & Feb. 4 - Mar. 31 & V & Bohr's atom \\
\hline n. 48 - n. 59: & Apr. 17 - May 15 & VI & Spectral multiplicity \\
\hline n. $60-$ n. 65: & $\begin{array}{l}\text { May 19 - Jun. 2 } \\
\text { (summary of the course) }\end{array}$ & & \\
\hline \hline
\end{tabular}

TABLE 2. Correspondence between the lectures of the course on Theoretical Physics Fermi held in 1927-28 and the first six chapters of his book 4 .

more phenomenological approach of Fermi was always latent and never abandoned by Majorana, as we shall see later. In those years, Majorana aimed at a full understanding of the novel Mechanics, based on solid and general mathematical grounds but without disregarding evidence coming from atomic and nuclear experiments.

3.1. Majorana's involvement with Physics teaching. Besides Majorana's Volumetti, his "Quaderni" ("Notebooks"), whose analysis has just began, record research studies up to approximately 1932 (see 9]). In those years, Majorana was used to frequent the Fermi group in Rome, although without even seeking for a stable position, which he would only obtain in Naples at the end of 1937. However, Majorana succeeded in obtaining the professorship degree of libero docente (an academic title analogous to the German Privatdozent) in January 1933 and, due to 
this position, he proposed some academic courses. The programs concerning three courses he would have given between 1933 and 1937 have only recently been discovered in the Archives of the University of Rome. This retrieval is very important and surprising, since these original documents cover a period that has been referred to as Majorana's gloomy years by the testimonies of that epoch (see, for example, [5], 6], 11]), during which he secluded himself from the life of the Institute.

The three programs are here translated in Appendixes B, C, D. Even at a first look, it is evident that Majorana was very careful in choosing the topics he would have treated in his proposed courses, and this reveals his genuine interest in advanced physics teaching.

In 1933, Majorana benefited from a C.N.R. visiting fellowship at the Institute of Theoretical Physics in Leipzig directed by Heisenberg. He went back to Italy for the Easter holidays between the end of April and the beginning of May, and his first program was submitted precisely at this time: the novel and stimulating atmosphere found in Leipzig probably had spurred him. He reiterated his request to give advanced Physics courses also in subsequent years, but he never delivered any course in Rome. This is implicitly testified by his friends and colleagues and explicitly confirmed by Majorana himself, who in the three programs declared he had not given any course before: a circumstance likely due to the lack of students.

The first two programs correspond to the courses in Mathematical Methods of Quantum Mechanics and Atomic Physics, respectively, and partially overlap. In presenting Quantum Mechanics, Majorana aimed to use group-theoretic methods: that was an unusual approach for the times, as already discussed [2, and will again be adopted in Majorana's lectures at the University of Naples in 1938. The approach will become the standard one in the teaching of Quantum Mechanics only many years after the Second World War.

The second program contains references to questions more phenomenological in nature and the third one deals with Quantum Electrodynamics: that was again an unusual topic for the Italian academic courses of the period, but had been fascinating Majorana for a long time, as clearly emerges from the unpublished research notes in his Quaderni.

3.2. Lecturing at the University of Naples. In 1937, the national competition for a full professorship in Theoretical Physics at the University of Palermo provided a good chance for entering advanced Physics teaching, for Majorana as well as for other skilful young physicists such as Gentile, Wick, Leo Pincherle, Giulio Racah, and Gleb Wataghin. Majorana was urged to take part in the competition by his colleagues and friends [5], 11. In particular, Fermi persuaded him to finally publish an earlier work of his, on the symmetrical theory of electrons and positrons 12, which will become famous for the appearance of the so-called 'Majorana neutrino' theory. Majorana had already faced (and solved) this problem long before, as clearly appears in his Quaderni. An explicit mention of "the positive electron and the symmetry of charges" was also recorded in the third program, which Majorana had submitted on April 28, 1936.

Without going through the details of the 1937 competition (already discussed in the literature [5], [6], [1]), we just recall that Majorana was eventually appointed as a full professor of Theoretical Physics at the University of Naples "for high and 
well-deserved repute, independently of the competition rules." He gave only twentyone lectures at the Institute od Physics in Naples, from January 13 to March 24, before his mysterious disappearance on March 26, 1938 [13, 14].

Fortunately, some manuscripts of the lecture notes written by Majorana himself for the students of his course on Theoretical Physics are available. He entrusted them to one of his students, Ms. Gilda Senatore, before he disappeared. They are deposited at the Domus Galilæana in Pisa and were reproduced in a book some years ago 13. However, a transcription comprising six lectures not included in the Pisa collection has recently been discovered [14. Therefore, in order to go through Majorana's teaching strategies, the analysis of his course should be updated (see 15]) and embrace some previously unknown topics, mainly Relativity (an account of this is in [2]).

Even if a comprehensive record of the course Majorana actually held in Naples in 1938 is not available, some peculiar - historical and scientific - features can be drawn from his very lecture notes.

In the prolusion, delivered on January 13, Majorana gave a broad outline of his course, aimed at the study of Quantum Mechanics and its applications to Atomic Physics (see Refs. 13, 2] and [15]). He also stated his teaching method, which would in fact consist of a combination of two: he mentioned the "mathematical method", through which "the quantum formalism is presented in its most general and therefore its clearest propositions from the very beginning and only afterwards are the criteria for applying it explained;" and he mentioned what he called the "historical method," which "explains how the first idea of the formalism appeared." Majorana regarded them as two opposite methods, but he aimed at a fruitful synergy between them.

It is really intriguing to note how closely Majorana's ideas resemble the preface to Paul A.M. Dirac's book on "The Principles of Quantum Mechanics." As for the mathematical presentation of quantum theory, Dirac wrote in 1930: "The symbolic method [...] deals directly in an abstract way with the quantities of fundamental importance," allowing the physical laws to be expressed "in a neat and concise way;" as for the applications, they "follow strictly from the general assumptions." However, all this implied "a complete break from the historical line of development" 16 .

We do not have any written notes of Majorana's lectures from the second to the fifth ones and we will speculate on their possible content in Section 5.

The remainder of the course is approximately divided into three parts:

- Old Quantum Mechanics (3+1/2 lectures);

- Electromagnetism, Relativity and their application to microscopic phenomena $(6+1 / 2$ lectures $)$;

- Mathematical foundations of Quantum Mechanics (6 lectures).

In the first of these parts Majorana is probably expanding on topics introduced in the first four lectures. He deals with the relevant phenomenology of Atomic Physics and its interpretation in the framework of the Old Quantum Theory of Bohr and Sommerfeld. In particular, starting from the available spectroscopic data, he introduces the spin and the related Pauli exclusion principle, accounting for the periodic table of the elements and the features of the spectra of one- and two-electron atoms in the Sommerfeld theory. 
The second part starts with the classical radiation theory, reporting explicit solutions of the Maxwell equations for a given system; some applications (e.g. scattering of light on the atmosphere) are discussed as well. Then Majorana deals with the Theory of Relativity, starting from simple phenomenology and later introducing the appropriate mathematical formalism. Lorentz transformations along with their immediate consequences are introduced in a simple and original way, and applications to the electromagnetic field are extensively deduced. Particular emphasis is given to the relativistic dynamics of electrons, which Majorana obtains from a variational principle.

The effort gone into Electromagnetism and Relativity ends up with the discussion of processes like the photoelectric effect, Thomson scattering and the Compton effect. The Franck \& Hertz experiment is also treated here, exhibiting the existence of energy levels. All these phenomena are considered as the phenomenological bases for Quantum Mechanics, and this appears quite 'in contrast' with the approach of the first part of the course, based on spectroscopic phenomenology.

The third set of lecture notes is more mathematical in nature. The (nonrelativistic) wave equation along with its statistical interpretation based on the Heisenberg uncertainty principle are presented only after a detailed discussion of some relevant topics on matrix and operator theory and Fourier transforms.

The original manuscripts collecting Majorana's lecture notes include a last discorsive part (see [13, 17, 15). It has been primarily interpreted as the notes of a lecture that Majorana would have held at the University of Naples if he did not mysteriously disappear. However, as already noted in [17, an accurate analysis of the content of this manuscript seems not to confirm a similar conclusion. In fact, the topics are rather advanced and do not relate, to any extent, to the last lecture (the 21st one) on the uncertainty principle. Moreover, the style of presentation of the topics is completely different from that used in the main body of the Naples lectures. Rather, the expository form resembles the style he had used in his prolusion.

\section{The progress of Majorana's approach to Theoretical Physics}

Majorana's approach to Theoretical Physics is well traced in his "Volumetti" 9]. Standard mathematical tools (differential equations, Fourier integrals and so on) dominate approximately the first half of these documents. They are used in order to solve well-defined physical problems, such as, for example, the heat propagation in an isotropic and homogeneous medium or the equilibrium of a rotating fluid (Clairaut's problem), or even to face contemporary problems such the scattering of an $\alpha$ particle by a radioactive nucleus or the solution of the Thomas-Fermi equation with the appropriate boundary conditions for atoms [18, [8].

Majorana's approach, however, sensibly changes in the remainder of his "Volumetti", dating to the end of 1928 and largely devoted to Group Theory and symmetries, which topics underlie almost all his articles. In this respect, an illuminating example is that of the infinite-dimensional unitary representations of the Lorentz group [2], whose study, reported in detail in the Volumetto $V$, forms the basis for his important article of 1932 [19] on a relativistic theory of particles with arbitrary spin.

An approach similar to the latter is also present in the three proposed programs, but some comments are needed here. 
From what reported in Appendix B, it is evident that the program on Mathematical Methods of Quantum Mechanics submitted in 1933 is entirely based on group-theoretic methods. Majorana mentions the elements of unitary geometry and transformations, pointing at the modifications to classical kinematics and at the invariance under transformation group. Then he goes through the rotation and permutation groups. The 'standard' presentation of Quantum Mechanics (both in the Schrödinger version and in the Heisenberg one) is completely removed from this program. Even the applications (e.g. angular momenta, systems with identical particles) are strictly related to the formalism of Group Theory. The same applies to the Theory of Relativity, mentioned in the framework of the Lorentz group and of spinor calculus.

Majorana's attitude towards transformation theory resembles what Dirac wrote in his preface about the formulation of the fundamental laws of Nature, which "requires the use of the mathematics of transformations. The important things in the world appear as the invariants [...] of these transformations." Further, almost all the topics mentioned in Majorana's 1933-34 program were explicitly treated by Weyl in his book: the item 1 in the program appears in the chapter 1 of Ref. [10, entitled precisely "Unitary Geometry"; the items from 3 (partially) to 7 of Majorana, mentioning representations and applications of Group Theory to momenta, identical particles, and relativistic particles, are also dealt with in chapters 3,4 and 5 of the Weyl book. An exception seems to be the discussion of the phase space and the introduction of the quantum of action in item 2, which does not appear to correspond to any topic in Weyl's book.

The proposed impressive use of Group Theory is certainly justified by Majorana's own attention to conciseness and generality, which is constantly present in his studies. We saw that Majorana himself explicitly stated this attention in the prolusion to his course, where he also followed Dirac. The latter gave up the usual "method of co-ordinates or representations" through his own preference for the "symbolic method." However, also the line of thinking followed by the Leipzig school and experienced by Majorana in 1933 [5, 11, just when he officially submitted his first program, may have played some role on Majorana's attitude to Group Theory.

Things are somewhat different in the 1935-36 program on Mathematical Methods of Atomic Physics (we recall no program is available relative to 1934-35). A more phenomenological approach emerges, compared with that proposed in 1933-34. The group-theoretic methods appear only marginal and subordinated to practical applications, like complex atomic spectra and hyperfine structures. It would seem quite evident that the choice of the items for this course was influenced by the line of thinking adopted by the Rome group headed by Fermi, who had been engaged in spectroscopic researches before switching to nuclear physics [20]. Further, in the program submitted by Majorana, the mention of the elements of Nuclear Physics precisely follows that of hyperfine structures. This sequence is in agreement with the course of events recalled by Segrè 6 in giving account of the switching from atomic to nuclear researches in Rome. ${ }^{4}$

On the contrary, the 1936-37 program of Quantum Electrodynamics appears free from external influences, probably due to the absence of Majorana from the Institute of Physics in those years [5]. The program is largely based on personal

\footnotetext{
${ }^{4}$ Note also that a contribution of Majorana is acknowledged in the paper by Fermi and Segrè on hyperfine structures [21].
} 
studies which are easily recognizable in his handwritten notes (the "Quaderni" and partially the "Volumetti").

A further elaboration by Majorana of his own teaching approach comes out when we compare the known lecture notes of the course he held in Naples with the three programs submitted in Rome. The phenomenological part of Majorana's 1938 course strictly resembles Fermi's 1927-28 course. The mathematical part, instead, has contact points with some items Majorana mentioned in his three programs. In particular, the unitary geometry (only loosely borrowed from the Weyl book) mentioned in the first program is then discussed in lecture n.17 of the Naples course; matrix calculus (second program) is then introduced in lecture n.16, and the relativistic theory of the electron (second and third programs) is expounded in lectures n.13 and n.14 [14]. This mathematical part appears as peculiar of Majorana's own teaching approach, and inspired by Weyl and the group-theoretic methods as well as by Dirac. It is quite distant from other contemporary approaches as, for example, those followed by E. Persico [22], whose book was all the same recommended to Majorana's students [14].

\section{Common traits in Fermi's and Majorana's Courses}

As is well-known, Old Quantum Theory essentially superimposes few simple rules on the conceptual scheme of Classical Mechanics. Its framework survived as a relevant tool in Theoretical Physics courses for many years, an intuitive representation of physical phenomena being preserved by it. It was only about the 1950 s that Quantum Mechanics could thoroughly get in at the university courses, mainly with its Wave-Mechanical version.

Fermi dealt with The New Quantum Mechanics in the final chapter of his 1928 book. However, he did not lecture on Wave Mechanics at all before his 1928-29 course in Theoretical Physics. In the following academic year, 1929-30, Wave Mechanics took a total of twelve lectures out of fifty-eight, comprising a brief mention of Perturbation Theory (two lectures) ${ }^{5}$

The Bohr and Sommerfeld quantization rules underpin also the first group of Majorana's lectures, up to February 3, 1938. Furthermore, Majorana went into Matrix Mechanics in Naples since March 8, 1938 (and even before it in the three programs he submitted in Rome); he also lectured on Wave Mechanics starting from March 17 until he disappeared.

We are now going through Majorana's way of treating atomic physics in the framework of Old Quantum Theory. The legacy of Fermi's course will be readily disclosed in Majorana's lectures by our analysis.

5.1. Phenomenological approach and theoretical analysis. At a first sight, Fermi and Majorana's approaches appear not easily related to each other. In fact, a detailed relativistic treatment of the fine structure in the hydrogen spectrum is reported in Majorana's lectures, whereas Fermi only gave the final formula along with a few comments.

\footnotetext{
${ }^{5}$ Fermi's record book of his 1928-29 course in Theoretical Physics is missing. However, we can infer that he devoted some lectures to Wave Mechanics since he asked about the Schrödinger equation of a candidate during an exam in October 1929 (Archives of the University of Rome "La Sapienza"). As for Fermi's record book of his 1929-30 course, it should be noted that it is kept in Pisa rather than in the Rome Archives [23].
} 
As for Fermi's way to the fine structure, he firstly wrote down the Newton equations for one electron in the Coulomb field of the nucleus and then put forward the conditions to fulfill in order to fit the spectroscopic terms. In other words, he attained the Bohr-Sommerfeld conditions as a generalization of empirical rules. He also dealt with the motion of the nucleus, recalling the slight difference between the even lines of the Pickering series of $\mathrm{He}^{+}$and the Balmer series of $H$. Fermi then remarked that a relativistic correction to the Bohr formula should be taken into account. Indeed, Old Quantum Theory predicted $n$ different values ranging from 1 to $n$ for the azimuth $k$ of an electron in the $n$ orbit. The value $k=1$ corresponded to the highest eccentricity, while $k=n$ to circular orbits. Now, higher eccentricity meant a closer approach to the nucleus and higher velocities at the perihelion. Therefore, the lower $k$ the larger the correction according to Old Quantum Theory. The final formula was:

$$
W_{n, k}=-\frac{2 \pi^{2} m Z^{2} e^{4}}{h^{2} n^{2}}-\frac{\pi^{4} m Z^{4} e^{8}}{c^{2} h^{4} n^{3}}\left(\frac{8}{k+\frac{1}{2}}-\frac{6}{n}\right)
$$

Fermi stressed that, in the framework of Quantum Mechanics (where the azimuthal quantum number $k$ ranged from 0 to $n-1$ ), the relativistic correction was different from the previous formula, but the additional spin correction made the old result still hold (we find here another case where two opposite corrections cancelled out each other, besides the case already mentioned of the Compton effect). Fermi expounded these topics in some paragraphs of the fifth chapter of his Introduzione. Their titles are as follows:

- Motion of the electron in the hydrogen atom;

- Hydrogen spectrum;

- Hydrogen energy levels;

- Spectrum of the ionized helium;

- The Sommerfeld condition;

- Hydrogen elliptic orbits.

They correspond to the lectures from the 28th (February 4) to the 34th (February 28 ), and to the 37th (March 8, 1928).

Summing up, we can say that Fermi's approach to atomic levels was 'from below', relying on mere phenomenological (Balmer series) and intuitive (velocity of the perihelion) arguments. In this respect, we may note that Fermi's intuitive and phenomenological approach appears as opposed to the more abstract approach theorized for example by Dirac, who wrote: "A book on the new physics, if not purely descriptive of experimental work, must be essentially mathematical."

Differently from Fermi, a very general method was used by Majorana in his known lectures to calculate the correction leading to the fine structure formula. Majorana wrote straight down the relativistic Hamiltonian and the Hamilton equations of motion for the electron in the hydrogen atom. Then he introduced the Sommerfeld conditions, leading in the end to the same formula written down by Fermi (just in a slightly different form):

$$
\nu_{n, \ell}=-\frac{W_{n, \ell}}{h}=Z^{2} \frac{R}{n^{2}}+Z^{4} \alpha^{2} \frac{R}{n^{3}}\left(\frac{1}{\ell}-\frac{3}{4 n}\right) .
$$


There is no echo of Fermi's intuitive and phenomenological approach. However, the crucial point is that Majorana's treatment of the relativistic corrections was not the way Majorana introduced the quantized atom. In his notes there are several references calling up various points that Majorana tackled in the four missing lectures, held before January 25, 1938. For example: "As we remember," the non relativistic expression for the radial momentum is analogous to the relativistic one; furthermore, "we already know" the value of the integral and the total quantum $n=n_{r}$ is introduced "here as well." Majorana also mentions the $\mathrm{He}^{+}$spectrum, in such a way that we may infer he had already dealt with it in the very first part of his course. In other words, it is very likely that Majorana introduced such topics as the Balmer series, the Sommerfeld conditions, and the elliptic orbits in his first four lectures, whose notes are not available. As a consequence, there is no evidence that his approach to the atom was substantially different from Fermi's one. All we can say is that Majorana dealt with the relativistic corrections in more details than Fermi, so confirming his attitude towards generalization. Although he roughly condensed the topics of the whole of Fermi's course in about ten lectures, this circumstance did not hinder him from providing a deep insight into Old Quantum Theory.

Once the relativistic correction was given, Majorana drew his attention to the motion of the nucleus (and differently from Fermi he also provided the formula for the reduced mass). He pointed out that the Rydberg constant could now agree with the value "already given." However, the empirical selection rules lacked of an underlying theoretical account, which Majorana characterized as one of the "difficulties" of the Sommerfeld theory of the atom; note that the selection principle and the mean life of the quantum states are further topics which follow the fine structure one in Fermi's book (Fermi also dealt with them in his 42th lecture on March 20, 1928).

5.2. A clear legacy. The strong analogy with Fermi's approach to the atom becomes more evident when Majorana deals with spatial quantization, alkali spectra, and spin. He shows that the degeneracy of the orbits can be resolved by applying a magnetic field to the atomic system. He still moves within the framework of what he himself called the "old theory," and he again uses the "elementary theory" in dealing with the Bohr magneton. The final formula for the splitted lines is explicitly reported:

$$
\nu=\frac{W(n, \ell, m)-W\left(n^{\prime}, \ell^{\prime}, m^{\prime}\right)}{h}=\frac{W(n, \ell)-W\left(n^{\prime}, \ell^{\prime}\right)}{h}+\left(m-m^{\prime}\right) \frac{\mu_{0} H}{h} .
$$

It is almost identical with the formula of the Zeeman effect reported by Fermi (Eq. (57) on page 179 of Ref. 4]). In fact, in the fifth chapter of his book Fermi also went into spatial quantization, Bohr magneton, the spinning electron and, in the end, the Zeeman effect and the Stark effect - corresponding to lectures from the 43th (March 22) to the 47th (March 31). Going back to Fermi's attitude for intuitive models, we also note that Fermi introduces the Lorentz theory of the Zeeman effect before the quantum treatment of the latter: although the early theory "has been replaced by the quantum theory [...] it accounts for many phenomena in a very simple way." Majorana instead does not mention the Lorentz theory nor the Stark effect, which one might ascribe perhaps to his already recalled need for brevity. 
Fermi's preference for intuition and simplicity is clearly stated at the head of the sixth chapter, devoted to spectral multiplicity: "We are going to exploit the Sommerfeld method to attain a more simple and intuitive picture of the atom." Fermi puts forward the distance-dependent shielding effect due to the inner electrons, and provides an explicit expression for the effective charge acting on the optical electron closely resembling the one later reported by Majorana. Here again, the smaller the azimuth, the closer the approach of the semiclassical orbit to the nucleus at the perihelion, the stronger the attractive force on the optical electron. Fermi provides some further details about the terms in a non Newtonian central field, illustrating the energy levels of sodium with a scheme. Now, the same scheme with the $\mathrm{Na}$ spectrum and the same phenomenological arguments accounting for the splitting of the lines are reported also by Majorana in his course.

Majorana's lecture of January 29, 1938 deals with Pauli's exclusion principle, giving an account of the periodic table, and the alkali metal spectra. The same topics were (more extensively) reported in the sixth chapter of Fermi's book, in the following paragraphs:

- Central motion in a non Newtonian central field;

- The series of the spectral terms;

- The spectra of the alkali metals;

- The inner quantum;

- The counting of the quantum states;

- The exclusion principle and the electronic rings;

- Chemical and spectroscopic applications.

These paragraphs roughly correspond to Fermi's lectures from the 48th (April 17) to the 54th (May 3).

Majorana's notes resemble the part of Fermi's course just mentioned not only for its general formulation, but also for many formal details. For example, they both report many common formulae. Fermi puts forward the deep similarity between the electronic configurations of alkali metals, and also of some analogous metals. As for the latter, Fermi explicitly mentions only the case of $C u, A g$, and $A u$, as also Majorana does. Furthermore, in dealing with the Stoner table, Fermi uses the symbol $C p$ for Cassiopeium, which at the time was the German version of the French-inspired name Lutetium for the element 71; Majorana, with the same notation, reports the symbol $C p$ in his Stoner table.

The most humble but, at the same time, the most eloquent example of similarity occurs with the exclusion principle: Fermi writes that "at most only one electron" exists with the given four quantum numbers. In an early version Majorana wrote that there is "only one electron" in one orbit, given the four quantum numbers. However, he then corrects his sentence specifying that there is "at most only one electron," exactly the same expression Fermi used. One may regard this as an emblem when describing the legacy of Fermi's course in Majorana's lectures. However, it should be kept in mind that this legacy does not concern only formal details but appears to involve also meaningful aspects such as the attention paid to phenomenological descriptions.

Once such strong correspondence is established, it is straightforward to find some other examples of it in the first part of Majorana's lecture devoted to The spectra of the atoms with two valence electrons (which concludes the first set of lectures we mentioned in the paragraph 3.2). It corresponds to Fermi's paragraph which 
follows the applications of Stoner's table, and to Fermi's lectures NN.55-56 (May $5-8,1928)$. One more topic common to both Fermi and Majorana's courses is the scattering of light from the atmosphere.

On Thursday February 17, 1938 Majorana dealt with further issues in the framework of Old Quantum Theory, lecturing on the Compton effect and the Franck \& Hertz experiment. We will report on them soon.

5.3. Majorana's first four lectures. Neither Majorana's personal notes nor their transcription cover the first lectures up to January 22, 1938. All the same, the very analysis of those notes brings out what the four lectures from January 15 to 22 should be concerned with. Majorana himself explicitly recalled some of the topics he had already dealt with in his first lectures: we have already mentioned the Balmer series, the Sommerfeld conditions, the elliptic orbits. The case can be readily added of the nomenclature of the energy levels: the meaning of the $s, p, d$, $f, \ldots$ levels was taken for given when Majorana dealt with the alkali metals and their spectra.

But what about the points which Majorana might deal with before January 25 without explicitly mentioning them in his subsequent notes? To this purpose, we can exploit the analogy with Fermi's course. In other words, if we take for granted that Majorana's course followed the main lines of Fermi's lectures for what concerned Old Quantum Theory, we can speculate about the points dealt with by Majorana from January 15 to January 22, 1938 by merely looking at the topics of the first chapters of Fermi's Introduzione.

Thus, some of the first topics tackled by Majorana might well be the kinetic theory of gases and statistical mechanics: they introduced Fermi's course into atomic models. Fermi also showed how the quantized levels of the harmonic oscillator could lead to the Planck formula. Here we have two more topics Majorana should deal with: a) As for the Planck theory, Majorana wrote down the Planck constant in his formulae but never made a word of its meaning nor of its value in his notes following January 22. b) As for the harmonic oscillator, it is worth noting that the zero point energy had been repeatedly touched by Fermi in his papers preluding to the quantum statistics [24. In the first chapter of his Introduzione, Fermi, in spite of being in the framework of Old Quantum Theory, remarked that the zero point energy provided a better agreement with the experiments [p. 129]. Now, it could be likely that Fermi actually mentioned the zero point energy in his course as well, and therefore that Majorana too touched that point, even if in dealing with Old Quantum Theory.

The Bohr-Sommerfeld theory, the Zeeman and the Stark effects, the selection rules and the correspondence principle are some more topics which Majorana likely borrowed from Fermi's course in his first four lectures.

\section{A STEP AHEAD}

6.1. About the missing notes. The question now arises: which were the fates of the notes concerning the four lectures from January 15 to January 22? It appears hard to answer, but a closer look reveals something more: maybe it is not a well posed question. The point is intriguing.

We know that on January 22, 1938 Majorana wrote from Naples to his mother in Rome: "I believe I will be there within a few days, but only for few hours, since I have to take a book from Treves [it was the name of a publishing house] and some 
more from home" (the text of the letter is reported in Ref. [1]). We recall that the notes we have begin with Majorana's lecture of Tuesday, January 25, 1938, the first day available after Saturday 22. Which were the books Majorana urgently needed to bring to Naples in those same days when he was giving his course? Is it by chance that his notes start only after the journey he announced to his mother? We suggest a possible outline: the course of 1938 was the first one Majorana had ever held. After a few lectures introducing Old Quantum Theory, he probably realized that some notes would be useful for his student. Some textbooks on Theoretical Physics would help him to write the notes down. Fermi's book would fit extremely well for what concerned Old Quantum Theory, since Majorana was very familiar with Fermi's 1927-28 course. Thus, our tentative answer is that the notes on the first four lectures, from January 15 to 22 , had no fate at all, simply because they had never been written down by Majorana.

An indirect check of the outline we have just suggested is as follows. As already mentioned, Majorana came back to the motion of the nucleus "in the framework of non relativistic mechanics." That sounds like a step backwards with respect to the treatment of relativistic corrections he had just concluded. However, his choice seems to fit very well with our idea that Majorana had just taken Fermi's book from Rome: maybe he had just realized that an interesting topic was left out in his previous lectures. ${ }^{6}$

6.2. Overtaking old positions. We have just seen that Fermi's phenomenological approach resounded in Majorana; in particular, it is through the latter's lectures on Old Quantum Theory that Fermi's legacy is revealed. However, the attention paid to phenomenology by a theoretician such as Majorana should not be regarded as obvious. For example, we have already mentioned that Dirac held that the educational approach to the new physics should be "essentially mathematical."

In fact, Dirac and Fermi can be regarded as two very different personifications of a theoretical physicist: more inclined to mathematical formalization the former, to the use of intuitive arguments the latter. These differences became conspicuous by Fermi's well-known formulation of the quantum theory of radiation, which he worked out between the end of 1920s and the beginning of 1930s since "the method used by Dirac did not appeal to him" [25]. ${ }^{7}$

The attention to a phenomenological and intuitive approach coexisted with a more mathematical formulation of quantum aspects in Majorana's courses, and his teaching attitude may be regarded as synthesizing Fermi's and Dirac's positions.

\footnotetext{
${ }^{6}$ The latter is not the only episode. Take the Franck \& Hertz experiment, which follows the Compton effect and precedes Matrix and Wave Mechanics in Majorana's notes: though the Compton effect appears as a direct application of the electromagnetism and the quantum theory of light, the experiment revealing the energy levels of mercury vapors appears rather out of context. Before the new transcription of the notes was discovered, the Franck \& Hertz experiment was considered part of "a series of relevant physical phenomena" which Majorana tackled "in order to put forward masses of physical facts and results in the simplest way [...] before he started the hard journey towards the new mechanics" (see N. Cabibbo, in Ref. [13 pp. 118-119). Now we know six more lectures and we have to account for the fact that the Franck \& Hertz experiment immediately follows a whole set of lectures on electromagnetism and relativity.

${ }^{7}$ Some placid rivalry possibly existed between them, dating back to the formulation of the Fermi-Dirac statistics 26. E. Rutherford himself appears to make fun of that when, in congratulating Fermi on his discovery of neutron-induced radioactivity, he added: "You may be interested to hear that Professor Dirac also is doing some experiments. This seems to be a good augury for the future of theoretical physics!" [27].
} 
Dirac was aware that mathematics alone might hide the physical meaning of theories, but simply he was not scared of it: "One should learn to hold the physical ideas in one's mind without reference to the mathematical form." On the other hand, Majorana considered that as a serious drawback: "The mathematical method thoroughly frustrates one's desire to intuit the physical meaning" of the formalism; not to mention that, according to him, the pre-existing mathematical tools "had been forced" by Quantum Mechanics to fulfill practical purposes.

Thus, an overtaking of Fermi's and Dirac's positions may be got through Majorana's teaching attitude and, more explicitly, through the prolusion to his course of 1938: the phenomenological presentation of the Old Quantum Theory, borrowed from Fermi's intuitive approach, was supplemented by the deepening of forefront mathematical aspects of Quantum Mechanics. At the same time, the limitations of a purely mathematical presentation - that sort of presentation to which Dirac was inclined - were put forward by Majorana, rising attention to more intuitive aspects connected with quantum theories and their historical lines of development.

\section{Conclusions}

In the present paper, we suggested a comparison between Fermi's and Majorana's attitudes towards the teaching of Theoretical Physics. Fermi's 1927-28 course on Theoretical Physics was crucial for our analysis. From Fermi's record book relative to his 1927-28 course, a strong correspondence emerges between the topics Fermi delivered in his lectures and six chapters of his 1928 book on Atomic Physics. This allowed a detailed reconstruction of the content of his lectures on Theoretical Physics, despite no written note being available on those lectures.

The influence of Fermi's lectures can be perceived in Majorana's personal notes dating back to the late 1920s. Further, Majorana's personal notes and the programs of three courses he submitted in Rome between 1933 and 1936 revealed his forefront approach and his search for new routes in dealing with Quantum Mechanics.

We carried out a detailed comparison between Fermi's 1927-28 course on Theoretical Physics and the one Majorana gave in Naples ten years later. On the basis of the available notes of Majorana's course we showed that a number of Majorana's lectures - those dealing with Old Quantum Theory - very closely reflect Fermi's 1927-28 course. That helped to conjecture about the content of some of Majorana's previous lectures, for which no written notes are available at all.

On the other hand, Fermi's approach just represented the starting point for further theoretical developments in the reminder of Majorana's course, devoted to the new Quantum Mechanics in both its wave mechanical and matrix forms. Majorana's mathematical approach, with the peculiar resort to Group Theory, proved his original teaching touch. In fact, a thorough difference with Fermi's lectures already emerged in the three programs submitted by Majorana at the University of Rome. Majorana's courses thus consisted in a fruitful mixture of an original approach - very similar to that of the present courses in Quantum Mechanics - and of some consolidated lines of development - which were the clear legacy of the lines Fermi had adopted in his courses.

In conclusion, we have evidence of a deep influence of Fermi's teaching approach on Majorana's one and, at the same time, a thorough autonomy between their scientific personalities. 


\section{Appendix A. Fermi's 1927-28 Course on Theoretical Physics}

The topics treated by Fermi in his lectures (labelled by their order number and date) are reported in the following for the course in Theoretical Physics at the University of Rome in 1927-28.

\begin{tabular}{|c|c|c|}
\hline N. & Date & Topics \\
\hline 1 & $(15 \mathrm{Nov})$ & Interactions between molecules. \\
\hline & & Solids and fluids in the framework of the kinetic theory. \\
\hline 2 & (17 Nov) & Dependence of pressure upon molecular kinetic energy. \\
\hline 3 & (19 Nov) & Mean free path. Vapor rays. \\
\hline 4 & (22 Nov) & Kinetic energy in general coordinates. \\
\hline & & The theorem of equipartition of the energy (without proof). \\
\hline 5 & (24 Nov) & Phase space. \\
\hline 6 & (26 Nov) & Computation of the probability of a distribution. \\
\hline 7 & (29 Nov) & The Boltzmann distribution law. \\
\hline 8 & $(1 \mathrm{Dec})$ & Proof of the theorem of equipartition of the energy. \\
\hline 9 & (3 Dec) & The Maxwell law. \\
\hline 10 & $(6 \mathrm{Dec})$ & Principles of vector field theory. \\
\hline 11 & (10 Dec) & Summary of the laws of electrology. Their differential form. \\
\hline 12 & (13 Dec) & Displacement currents. The Maxwell laws. \\
\hline 13 & (15 Dec) & $\begin{array}{l}\text { Propagation of plane electromagnetic perturbations. } \\
\text { Their velocity. }\end{array}$ \\
\hline 14 & $(17 \mathrm{Dec})$ & Polarized sinusoidal plane wave. \\
\hline 15 & (20 Dec) & Propagation of electromagnetic perturbations of arbitrary shape. \\
\hline 16 & (22 Dec) & Poynting vector. \\
\hline 17 & (10 Jan) & Electromagnetic moment and radiation pressure. \\
\hline 18 & (12 Jan) & Electronic theory of dispersion. \\
\hline 19 & (14 Jan) & Electromagnetic radiation theory. \\
\hline 20 & (17 Jan) & The electron. \\
\hline 21 & (19 Jan) & Positive nuclei and the Rutherford atomic model. \\
\hline 22 & (21 Jan) & $\alpha_{-}, \beta-$, and $\gamma$-rays. \\
\hline 23 & (24 Jan) & Radioactive transmutations. \\
\hline 24 & (26 Jan) & Light quanta. \\
\hline 25 & (28 Jan) & Compton effect. \\
\hline 26 & (31 Jan) & Bohr's hypothesis. \\
\hline 27 & $(2 \mathrm{Feb})$ & Energy levels. \\
\hline 28 & (4 Feb) & Kepler motions. \\
\hline 29 & (7 Feb) & $\begin{array}{l}\text { Dependence of the energy and the period on the orbital } \\
\text { elements of motion. }\end{array}$ \\
\hline 30 & (9 Feb) & Balmer series and the energy levels of hydrogen. \\
\hline 31 & (11 Feb) & Orbits of the stationary states. \\
\hline 32 & (14 Feb) & Determination of the Rydberg constant. \\
\hline 33 & (25 Feb) & The spectrum of $\mathrm{He}^{+}$and the motion of the nucleus. \\
\hline 34 & (28 Feb) & Mechanical properties of the stationary orbits of hydrogen. \\
\hline 35 & (1 Mar) & Sommerfeld conditions for multi-periodic systems. \\
\hline 36 & (6 Mar) & $\begin{array}{l}\text { Application of the Sommerfeld conditions to the oscillator } \\
\text { and the rotator. }\end{array}$ \\
\hline
\end{tabular}




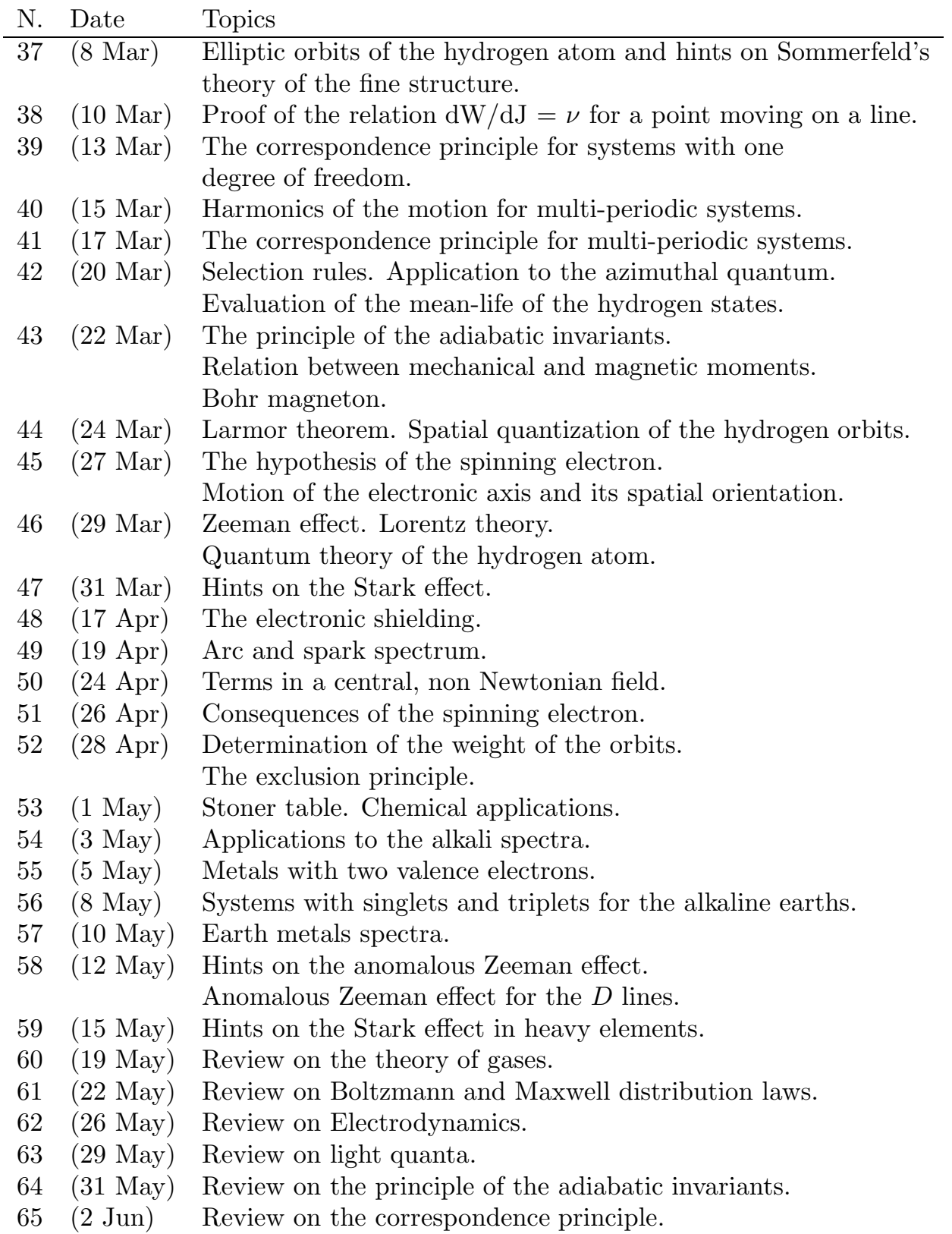




\section{Appendix B. Program of Majorana's first COURSe}

Title: Mathematical Methods of Quantum Mechanics

Academic Year: 1933-34

\section{Topics to be covered:}

1) Unitary geometry. Linear transformations. Hermitian operators. Unitary transformations. Eigenvalues and eigenvectors.

2) Phase space and the quantum of action. Modifications to classical kinematics. General framework of Quantum Mechanics.

3) Hamiltonians which are invariant under a transformation group. Transformations as complex quantities. Non compatible systems. Representations of finite or continuous groups.

4) General elements on abstract groups. Representation theorems. The group of spatial rotations. Symmetric groups of permutations and other finite groups.

5) Properties of the systems endowed with spherical symmetry. Orbital and intrinsic momenta. Theory of the rigid rotator.

6) Systems with identical particles. Fermi and Bose-Einstein statistics. Symmetries of the eigenfunctions in the center-of-mass frames.

7) The Lorentz group and the spinor calculus. Applications to the relativistic theory of the elementary particles.

Date of submission: May, 1933

\section{Appendix C. Program of Majorana's Second course}

Title: Mathematical methods of Atomic Physics

Academic Year: 1935-36

\section{Topics to be covered:}

Matrix calculus. Phase space and the correspondence principle. Minimal statistical sets or elementary cells. Elements of the quantum dynamics.

Statistical theories. General definition of symmetry problems. Representations of groups. Complex atomic spectra. Kinematics of the rigid body. Diatomic and polyatomic molecules.

Relativistic theory of the electron and the foundations of electrodynamics. Hyperfine structures and alternating bands. Elements of Nuclear Physics.

Date of submission: April 30, 1935

\section{Appendix D. Program of Majorana's third course}

Title: Quantum electrodynamics

Academic Year: 1936-37

\section{Topics to be covered:}

Relativistic theory of the electron. Quantization procedures. Field quantities defined by commutability and anticommutability laws. Their kinematical equivalence with sets with an undetermined number of objects obeying the Bose-Einstein or Fermi statistics, respectively. Dynamical equivalence.

Quantization of the Maxwell-Dirac equations. Study of the relativistic invariance. The positive electron and the symmetry of charges. 
Several applications of the theory. Radiation and scattering processes. Creation and annihilation of opposite charges. Collisions of fast electrons.

Date of submission: April 28, 1936

\section{REFERENCES}

[1] E. Segré, Enrico Fermi, physicist, University of Chicago Press, Chicago, 1970.

[2] A. Drago and S. Esposito, "Following Weyl on Quantum Mechanics: the Contribution of Ettore Majorana," Found. Phys., 34 (2004), 871-887. arXiv:physics/0503084.

[3] E. Fermi, Lezioni di Fisica teorica dettate dal Prof. E. Fermi, raccolte dai Dott. Dei e Martinozzi, [s.n.], Roma, 1927.

[4] E. Fermi, Introduzione alla fisica atomica, Zanichelli, Bologna, 1928.

[5] E. Amaldi, La Vita e l'Opera di E. Majorana, Accad. Naz. dei Lincei, Roma, 1966; Ettore Majorana: Man and scientist, in Strong and Weak Interactions: present problems, edited by A. Zichichi, New York and London, 1966.

[6] E. Segré, A mind always in motion. The Autobiography of Emilio Segré, University of California Press, Berkeley and Los Angeles, 1993.

[7] F. Rasetti in: E. Fermi, Collected Papers (Note e Memorie), edited by E. Amaldi et al., University of Chicago Press - Accad. Naz. dei Lincei, Chicago - Roma, 1962-1965, 2 vols., Vol. I (1962), p. 277.

[8] E. Di Grezia and S. Esposito, "Fermi, Majorana and the Statistical Model of Atoms," Found. Phys., 34 (2004), 1431-1450. arXiv:physics/0406030.

[9] S. Esposito, E. Majorana jr, A. van der Merwe and E. Recami (eds.) Ettore Majorana: Notes on Theoretical Physics, Kluwer, New York, 2003.

[10] H. Weyl, Gruppentheorie und Quantenmechanik (first edition), Hirzel, Leipzig, 1928. English translation from the second German edition in The Theory of Groups and Quantum Mechanics, Dover, New York, 1931.

[11] E. Recami, Il caso Majorana, Oscar Mondadori, Milano, 1991, 2nd edition; idem, Di Renzo, Roma, 2002, 4th revised and enlarged edition. See also E. Recami, "Ettore Majorana: l'opera scientifica edita e inedita," Nuovo Sagg., 3-4 (1999), 14-29; "Ricordo di Ettore Majorana a sessant'anni dalla sua scomparsa: l'opera edita e inedita", Quad. Storia Fis., 5 (1999), 19-68 arXiv:physics/9810023.

[12] E. Majorana, "Teoria simmetrica dell'elettrone e del positrone," Nuovo Cim., 14 (1937), 171-184.

[13] E. Majorana, Lezioni all'Università di Napoli, Bibliopolis, Napoli, 1987.

[14] S. Esposito, "Il corso di Fisica teorica di Ettore Majorana: il ritrovamento del documento Moreno," Nuovo Sagg., 21 (2005), 21-33; A. Drago and S. Esposito, "Ettore Majorana's course on Theoretical Physics: a recent discovery," e-print arXiv:physics/0503084

[15] E. Majorana, Lezioni di Fisica Teorica, edited by S. Esposito, Bibliopolis, Napoli, 2006 (in press).

[16] P.A.M. Dirac, The Principles of Quantum Mechanics, Clarendon Press, Oxford, 1930, p. viii.

[17] S. Esposito, "Una lezione particolare di Ettore Majorana," Quad. Storia Fis., to be published arXiv:physics/0512174.

[18] S. Esposito, "Majorana solution of the Thomas-Fermi equation," Am. J. Phys. 70 (2002) 852-856.

[19] E. Majorana, "Teoria relativistica di particelle con momento intrinseco arbitrario," Nuovo Cim., 9 (1932), 335-344.

[20] Concerning the path towards nuclear researches in Rome, see for example A. De Gregorio, "A Far-reaching project behind the discovery of neutron-induced radioactivity," Studies Hist. Phil. Mod. Phys., to be published.

[21] E. Fermi and E. Segré, "Zur Theorie der Hyperfeinstrukturen," Zeit. Phys., 82 (1933), 729749; "Sulla teoria delle strutture iperfini," Mem. Accad. d'Italia, 4 (1933), 131-158; reproduced in E. Fermi, Collected Papers, cit. in Ref. 7, Vol. I, pp. 514-537.

[22] E. Persico, Fondamenti della meccanica atomica, Zanichelli, Bologna, 1936. 
TEACHING THEORETICAL PHYSICS: THE CASES OF E. FERMI AND E. MAJORANA 21

[23] See M. Leone, N. Robotti, C.A. Segnini, "Fermi Archives at the Domus Galilaeana in Pisa", Physis, 27 (2000) 501-533

[24] L. Belloni, "On Fermi's route to Fermi-Dirac statistics," Eur. J. Phys., 15 (1994), 102-109; F. Cordella and F. Sebastiani, "I due lavori che preludono alla statistica quantica," Giornale di Fisica, 41 (2000), 83-101; "Sul percorso di Fermi verso la statistica quantica," Nuovo Sagg., 16 (2000), 11-22.

[25] E. Amaldi in: E. Fermi, Collected Papers, cit. in Ref. 7], Vol. I, p. 305.

[26] See for example the letter Fermi wrote to Dirac in 1926; in H. Kragh, Dirac: a scientific biography, Cambridge University Press, Cambridge, 1990, p. 36

[27] See E. Fermi, Collected Papers, cit. in Ref. 7, Vol. I, p. 641.

A. De Gregorio: Dipartimento di Fisica, Università degli Studi di Roma "La Sapienza", P.LE Aldo Moro, 00185 Rome, Italy (Alberto.DeGregorio@roma1.infn.it)

S. Esposito: Dipartimento di Scienze Fisiche, Università di Napoli "Federico II" \& I.N.F.N. Sezione di Napoli, Complesso Universitario di M. S. Angelo, Via Cinthia, 80126 NAPLES, ITALY (Salvatore.Esposito@na.infn.it) 